\title{
Hybrid Beamforming Design for Full-Duplex Millimeter Wave Communication
}

\author{
K. Satyanarayana, Student Member, IEEE, Mohammed El-Hajjar, Senior Member, IEEE, Ping-Heng Kuo, Alain \\ Mourad, Lajos Hanzo, Fellow, IEEE
}

\begin{abstract}
Harnessing the abundant availability of spectral resources at millimeter wave (mmWave) frequencies is an attractive solution to meet the escalating data rate demands. Additionally, it has been shown that full-duplex (FD) communication has the potential of doubling the bandwidth efficiency. However, the presence of significant residual self-interference (SI), which is especially more pronounced at mmWave frequencies because of the non-linearities in the hardware components, erodes the full potential of FD in practice. Conventionally, the residual SI is canceled in the baseband using digital processing with the aid of a transmit precoder. In this work, we propose a hybrid beamforming design for FD mmWave communications, where the SI is canceled by the joint design of beamformer weights at the radio-frequency ( $R F$ ) and the precoder as well as combiner in the baseband. Our proposed design preserves the dimensions of the transmit signal, while suppressing the SI. We demonstrate that our joint design is capable of reducing the SI by upto $30 \mathrm{~dB}$, hence performing similarly to the interference-free FD system while being computationally efficient. Our simulation results show that the proposed design significantly outperforms eigen-beamforming.
\end{abstract}

Index Terms-Full-Duplex, Millimeter Wave, MIMO, Beamforming, Hybrid Precoding.

\section{INTRODUCTION}

G IVEN the escalating mobile data rate demands, the world is pacing toward the next generation of wireless communication while also aiming for higher spectral efficiency. Recently, millimeter wave (mmWave) communication has gained much attention as one of the key disruptive technologies to deliver the ever-increasing data rates [1]. Since the spectral resources in the sub- $6 \mathrm{GHz}$ bands are already crowded, harnessing the wide bandwidths available at mmWave frequencies can significantly increase the capacity to accommodate a large number of users [1]. Additionally, it has been shown that full-duplex (FD) communication has the potential to double the communications spectral efficiency [2]-[4]. Therefore, the efficient employment of FD techniques at mmWave frequencies would further improve the network's spectral efficiency.

In mmWave communication, owing to its high propagation losses imposed by the attenuation due to foliage, atmospheric absorption and rain-induced fading, the signal-to-noise ratio (SNR) at the receiver would be typically low [5]. Therefore, to mitigate the propagation losses, directional transmissions are employed using large antenna arrays [6]. Given that wavelength at mmWave frequencies is on the order of few millimeters, large antenna arrays can be packed into a compact

The fiscal support of InterDigital as well as that of the European Research Council, Advanced Fellow Grant is gratefully acknowledged. area to attain high beamforming gains. Traditionally, beamforming at lower frequencies has been carried out using digital processing, which is not a convenient solution for mmWave frequencies, since it would require a dedicated radio-frequency (RF) chain for each and every antenna element. Hence, directional transmission at mmWave frequencies is employed using hybrid beamforming ( $\mathrm{HBF}$ ), in order to circumvent the hardware complexity and the need for power hungry analogto-digital/digital-to-analog converters (ADCs/DACs) dedicated to all of the RF chains [7]. The HBF is performed by cascading a digital precoder in the baseband and an analog phase shifter at the RF. The analog beamforming carried out at the RF stage is typically inaccurate because of the limited resolution of the phase shifters. The state-of-the-art HBF architectures include fully-connected HBF and sub-array-connected HBF [8], [9], where the signals are precoded in the baseband and phaseshifted at the RF stage and then fed to the transmit antennas. Most of the contributions in hybrid mmWave systems are focused on precoding/codebook designs for the RF and the baseband. To elaborate further, by exploiting the sparse nature of the mmWave channel, El Ayach et al. [10] proposed a hybrid precoder design based upon a popular technique known as orthogonal matching pursuit (OMP). In [11], Alkhateeb et al. proposed an RF codebook design for hybrid systems. A heuristic algorithm devised by exploiting channel's reciprocity was advocated by Eltayeb et al. [12]. Considering the hardware complexity and system performance, Chen [13] proposed an iterative hybrid beamforming, where phase shifters can only supply discrete phase adjustments while maximizing the spectral efficiency.

On the other hand, it is generally assumed that bidirectional radio communication in the same frequency band is not possible because of the high self-interference that results from the transmitter's own transmission at the local receiver. However, the research conducted in [2], [3] proposed a solution to this challenge, where simultaneous transmission and reception in the same frequency were designed, which is termed as full-duplex (FD) wireless communication. Henceforth, the FD communication philosophy has attracted the attention of the wireless communication researchers, given its potential to double the spectral efficiency. However, the significant amount of self-interference (SI) at the local receiver tends to prevent FD communication from attaining satisfactory performance gains. Typically, the SI cancellation at the local receiver is carried out by a combination of passive and active methods [2], [14]. The passive methods aim for increasing the path loss of the interfering signal through antenna isolation so as to reduce the SI power [15]. By contrast, the active methods rely on the knowledge of the transmitted signal and they are generally 
carried out in three different approaches [16], namely radiofrequency (RF) cancellation, antenna cancellation, and digital cancellation. In the RF cancellation, the known transmitted signal at the local transmitter is used as a reference signal in the RF chain to reconstruct and then to subtract the SI. In the antenna cancellation, two replicas of the transmitted signal having opposite phases are generated using multiple transmit and receive antennas and the SI is removed by adding the two opposite replicas [17]. In contrast to RF and antenna cancellation, digital cancellation is typically employed together with the RF or antenna cancellation to further suppress the SI in the baseband [17], [18]. However, the benefits obtained by cascading RF/digital cancellation to remove the SI may remain limited because of the distortions at various stages, such as the power amplifier non-linearity, I/Q imbalance, and phase noise [4]. This is especially more pronounced in mmWave communications because of the high non-linearities of the ADCs/DACs at mmWave frequencies [19]. Hence, considering these non-linearities caused by the ADCs/DACs, power amplifiers, I/Q imbalance and phase noise, the residual SI would be significantly high. In the state-of-the-art contributions on the FD, most of the work has been focused on SI cancellation for MIMO relay systems [20]-[24], while some other related work includes dynamic resource allocation for FD systems [25]. More recently, Wang et al. [26] has derived a closed-form expression for achievable rates for FD MIMO relay systems over Rician fading when linear receivers are employed. Considering the limitations of the input circuitry, Day et al. derived upper and lowers bounds on achievable sum rate for FD MIMO in [27]. Everett et al. [28] studied the performance analysis of passive self-interference suppression for FD, where the authors demonstrate that as high as $70 \mathrm{~dB}$ of SI suppression is possible in certain environments.

Although mmWave frequencies can provide large contiguous bandwidths, the efficient use of spectral resources is crucial to meet the escalating data rate demands. Hence, the efficient employment of FD communication at mmWave frequencies would further improve the attainable spectral efficiency. As far as FD communication is concerned, there is a paucity of literature on mmWave communications relying on FD techniques. Additionally, employing beamforming would further mitigate the SI. More recently, Xiao et al. [16] proposed a beamforming based FD for mmWave communication, where the authors designed transmit and receive beamforming weights to cancel the SI. In [29], Snow et al. demonstrated that SI cancellation of upto $40 \mathrm{~dB}$ using digital beamforming can be achieved relying on multiple coordinated transceivers. Despite the application of both the analog and digital cancellations, there is still a significant residual SI. Therefore, the SI cancellation relying on beamforming techniques is crucial at mmWave frequencies in addition to the conventional analog and digital SI suppression methods in the face of the strongly non-ideal nature of the ADCs/DACs and power amplifiers, which result in high residual SI [19]. Unfortunately, the designs in [16], [29] cannot be easily extended to HBF systems in mmWave communications, given that they are proposed for a single RF chain and an attempt to extend them to multiple RF chains becomes mathematical intractable. Hence, in this paper, we propose a $\mathrm{HBF}$ design, where the RF beamformer and baseband precoder are obtained from the fully-digital precoder. Our contributions are summarized as follows.

1) Against this background, in this paper, we consider FD communication at mmWave frequencies relying on hybrid beamforming, where we aim for mitigating the SI by jointly designing the transmit and receive RF beamformer weights and the precoder as well as combiner matrices. To design the beamformer, precoder and combiner matrices, we first obtain the fully-digital solution, where we resort to an iterative algorithm relying on the idealized simplifying assumption of having perfect channel state information (CSI). Then we derive the HBF solution from the obtained digital solution using least-squares approximation [30]. The proposed solution preserves the signal's dimensionality ${ }^{1}$, while mitigating the SI. Based on our simulation results, we show that the proposed design is capable of reducing the SI by upto $30 \mathrm{~dB}$ and hence performing similarly to the interference-free FD system.

2) We present the mathematical proof for the convergence of the proposed iterative design, where the objective function is minimized in each iteration. Furthermore, we show that the value of the objective function reduces in every iteration and it is lower-bounded by zero.

3) We present quantitative comparisons of our proposed design through simulation results, where we demonstrate that our proposed design achieves better performance gains than eigen beamforming, especially when the SI power is high.

The rest of the paper is organized as follows. In Section II, we detail the system model of FD in mmWave communication, while in Section III we present our proposed hybrid beamforming design to suppress the SI. Section IV and Section V discuss our simulation results, followed by our conclusions.

Notations: We use upper case boldface, $\mathbf{A}$, for matrices and lower case boldface, a, for vectors. We use $(.)^{T},(.)^{H},\|.\|_{\mathrm{F}}, \operatorname{Tr}(.) \mathbb{E}($.$) for the transpose, Hermitian$ transpose, Frobenius norm, trace and expectation operator, respectively. We adopt $\mathbf{A}(m, n)$ to denote $m^{\text {th }}$ row and $n^{\text {th }}$ column of the $\mathbf{A}, \mathbf{I}_{N}$ is the identity matrix of size $N \times N$, and $\mathbf{A} \succ 0$ indicate that $\mathbf{A}$ is a positive definite matrix. Finally, we use $\mathcal{C N}, \mathcal{U}$, and i.i.d. to denote complex-valued normal distribution, uniform distribution, and independent and identical distribution, respectively.

\section{SySTEM MODEL}

In this paper we focus on a point-to-point communication in order to show the precoder and combiner design in details ${ }^{2}$. The transmitter and the receiver of node $j$ are equipped with $N_{t}^{(j)}$ and $N_{r}^{(j)}$ antennas as well as by $N_{r_{(j)}}^{\mathrm{RF}}$ and $N_{t_{(j)}}^{\mathrm{RF}} \mathrm{RF}$ chains, respectively. The transmitter of node $j$ maps the bits

\footnotetext{
${ }^{1}$ In contrast to spatial suppression [31], where the signal is projected into the null space of the interference, which may result in a reduced signal dimension, our design preserves the dimensionality of the signal subspace where the rank of the received signal is equal to the number of signal streams transmitted.

${ }^{2}$ In a subsequent paper we are showing how the system would work with multiple nodes (users $>2$ ).
} 
to QAM symbols and employs both RF beamforming and precoding using the matrices $\mathbf{F}_{\mathrm{RF}}^{(j)}$ and $\mathbf{F}_{\mathrm{BB}}^{(j)}$ of size $N_{t}^{(j)} \times N_{t_{(j)}}^{\mathrm{RF}}$ and $N_{t_{(j)}}^{\mathrm{RF}} \times N_{s}^{(j)}$, respectively. The beamformed symbols are transmitted over the mmWave channel. Then, the receiver at the node $i$ combines the signal using the RF combiner $\mathbf{W}_{\mathrm{RF}}^{(i)}$ and baseband combiner $\mathbf{W}_{\mathrm{BB}}^{(i)}$ of sizes $N_{r}^{(i)} \times N_{r_{(i)}}^{\mathrm{RF}}$ and $N_{r_{(i)}}^{\mathrm{RF}} \times N_{s}^{(j)}$, respectively. Then, the received signal vector $\mathbf{y}^{(i)}$ at the receiver of node $i$ after RF and baseband combining is given $b^{3}$

$$
\begin{aligned}
\mathbf{y}^{(i)} & =\underbrace{\mathbf{W}_{\mathrm{BB}}^{H^{(i)}} \mathbf{W}_{\mathrm{RF}}^{H^{(i)}} \mathbf{H}_{\mathrm{ji}} \mathbf{F}_{\mathrm{RF}}^{(j)} \mathbf{F}_{\mathrm{BB}}^{(j)} \mathbf{s}^{(j)}}_{\text {desired signal }} \\
& +\underbrace{\mathbf{W}_{\mathrm{BB}}^{H^{(i)}} \mathbf{W}_{\mathrm{RF}}^{H^{(i)}} \mathbf{H}_{\mathrm{SI}}^{(i)} \mathbf{F}_{\mathrm{RF}}^{(i)} \mathbf{F}_{\mathrm{BB}}^{(i)} \mathbf{s}^{(i)}}_{\text {SI }}+\underbrace{\mathbf{W}_{\mathrm{BB}}^{H^{(i)}} \mathbf{W}_{\mathrm{RF}}^{H^{(i)}} \mathbf{n}^{(i)}}_{\text {noise }},
\end{aligned}
$$

where $\mathbf{n}$ is the noise vector of dimension $N_{r}^{(i)}$ whose elements are i.i.d. with distribution $\mathcal{C N}\left(0, \sigma^{2}\right)$, $\mathbf{s}^{(j)}$ is the signal vector of dimension $N_{s}^{(j)}, \mathbf{H}_{j i}$ is the mmWave channel spanning from the transmitter in the $j^{\text {th }}$ node to the receiver in the $i^{\text {th }}$ node, whenever $i \neq j$ and it is given by [32]

$$
\mathbf{H}_{j i}=\sqrt{\frac{N_{r}^{(i)} N_{t}^{(j)}}{N_{c} N_{\text {ray }}}} \sum_{n_{c}=1}^{N_{c}} \sum_{n_{\mathrm{p}}=1}^{N_{\mathrm{p}}} \alpha_{j i}^{\left(n_{\mathrm{p}}, n_{c}\right)} \mathbf{a}_{r}\left(\phi_{j i}^{n_{\mathrm{p}}, n_{c}}\right) \mathbf{a}_{t}^{T}\left(\theta_{j i}^{n_{\mathrm{p}}, n_{c}}\right) .
$$

To elaborate further, $\mathbf{H}_{j i}$ is the statistical channel matrix

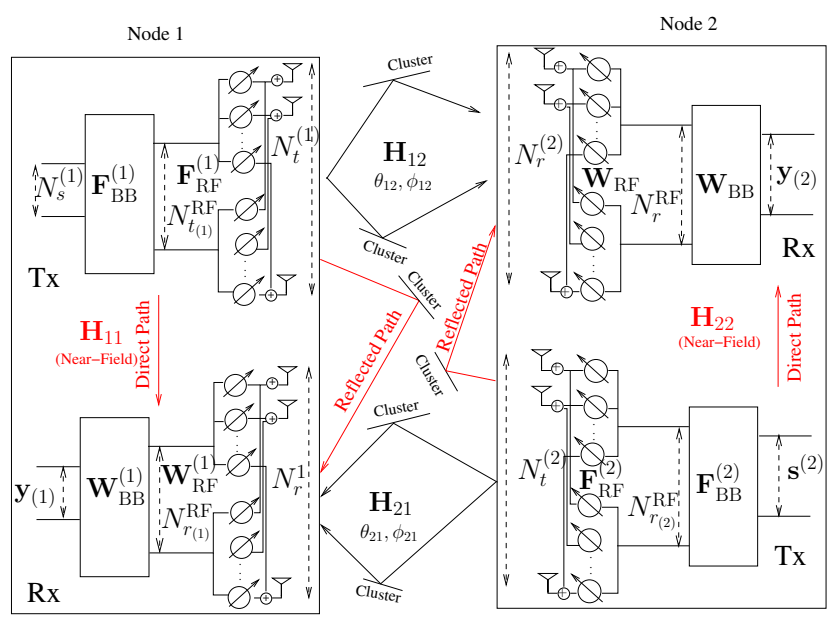

Fig. 1. Full-duplex with hybrid beamforming in mmWave communications.

of size $N_{r}^{(i)} \times N_{t}^{(j)}$ so that $\mathbb{E}\left[\left\|\mathbf{H}_{\mathrm{ji}}\right\|_{F}^{2}\right]=N_{t}^{(j)} N_{r}^{(i)}$, while $\alpha_{n_{c}}^{n_{\mathrm{p}}} \sim \mathcal{C N}(0,1)$ is a complex-valued Gaussian random variable, whose amplitude and phase are Rayleigh and uniform distributed, respectively. For a uniform linear array (ULA) having $N_{r}$ and $N_{t}$ antenna elements the response vectors $\mathbf{a}_{r}$ and $\mathbf{a}_{t}$ are expressed as:

$$
\begin{aligned}
& \mathbf{a}_{r}\left(\phi_{r}\right)=\left[1 e^{j \frac{2 \pi}{\lambda} d \cos \left(\phi_{r}\right)} \ldots e^{j \frac{2 \pi}{\lambda}\left(N_{r}-1\right) d \cos \left(\phi_{r}\right)}\right]^{T}, \\
& \mathbf{a}_{t}\left(\theta_{t}\right)=\left[1 e^{j \frac{2 \pi}{\lambda} d \cos \left(\phi_{t}\right)} \ldots e^{j \frac{2 \pi}{\lambda}\left(N_{t}-1\right) d \cos \left(\phi_{t}\right)}\right]^{T} .
\end{aligned}
$$

\footnotetext{
${ }^{3}$ The signal model considered is valid after both analog and digital SI cancellation.
}

Finally, $\theta_{t}$ and $\phi_{r}, N_{c}$ and $N_{\mathrm{p}}$ are the angles of departure (AOD) and arrival (AOA), and the number of clusters and rays, respectively.

For uniform planar arrays (UPA) having $N_{x}$ and $N_{y}$ antennas in the $x y$ plane, the response vector is given by

$$
\begin{aligned}
\mathbf{a}(\theta, \psi)= & {\left[1 e^{j \frac{2 \pi}{\lambda} d n_{x} \sin (\psi) \sin (\theta)+n_{y} \cos (\theta)}\right.} \\
& \left.\ldots e^{j \frac{2 \pi}{\lambda}\left(N_{x}-1\right) \sin (\psi) \sin (\theta)+\left(N_{y}-1\right) \cos (\theta)}\right]^{T},
\end{aligned}
$$

where $\psi, \theta$ are the elevation (AoA/AoD) and azimuthal angles (AoA/AoD), respectively.

Note that the node $i$ is operating in FD mode and hence can transmit at the same time, while receiving the signal from node $j$. Hence, the second term in (1) is the interference due to its own transmission. The matrix $\mathbf{H}_{\mathrm{SI}}^{(i)}$ is the near-field channel of node $i$ and is given as

$$
\mathbf{H}_{\mathrm{SI}}^{(i)}=\sqrt{\frac{\kappa}{\kappa+1}} \mathbf{H}_{\mathrm{ii}}+\sqrt{\frac{1}{\kappa+1}} \mathbf{H}_{r p},
$$

where $\kappa$ is the Rician factor, $\mathbf{H}_{\mathrm{ii}}$ is the near-field channel's lineof-sight (LOS) component (direct path as shown in Fig. 1) and $\mathbf{H}_{r p}$ is the reflected path of the self-interference. Fig. 2 shows the arrangement of the transmit and receive antenna arrays, where the arrays are separated by a distance $D$ at an angle $\Theta$. To elaborate further, in FD since the transmitter and the local receiver antenna arrays are closely spaced, where the distance $D$ shown in Fig. 2 would be typically few wavelengths, if the transmitter and the local receiver do not share the same array, and $D$ is zero if they share the same array [16]. Hence, the

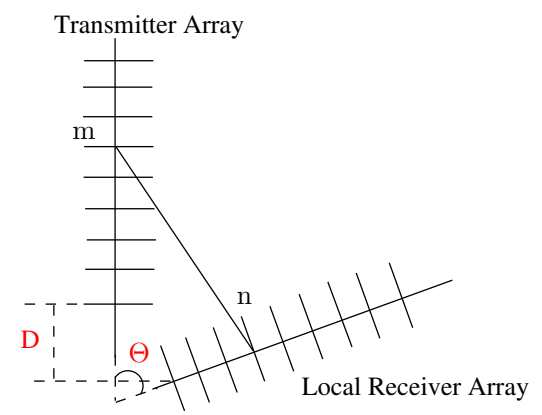

Fig. 2. The configuration of the transmitter and the local receiver antenna arrays of a node.

planar wave assumption does not hold, as it violates the farfield condition, i.e. $R_{o} \geq 2 \mathcal{D}^{2} / \lambda$ where $\mathcal{D}$ is the diameter of the antenna aperture as shown in Fig. 3. Typically, in the farfield as shown in Fig. 3 (a), the underpinning assumption is that the signal impinges on the antenna array as a planar wave, which means that all the antenna elements (AEs) observe the same path loss in the received signal and the phase difference between the adjacent antenna elements depends only on the angle of arrival and AEs spacing [33]. By contrast, in the FD scenario, since the transmitter and the local receiver are closely placed, these assumptions do not hold for the SI channel, as it is a near-field channel, which is shown in Fig. 3 (b). Therefore, a more realistic channel model, which is the spherical wave propagation model is considered for the nearfield LOS channel matrix $\mathbf{H}_{\mathrm{ii}}^{(i)}$ [34] and the channel coefficient 


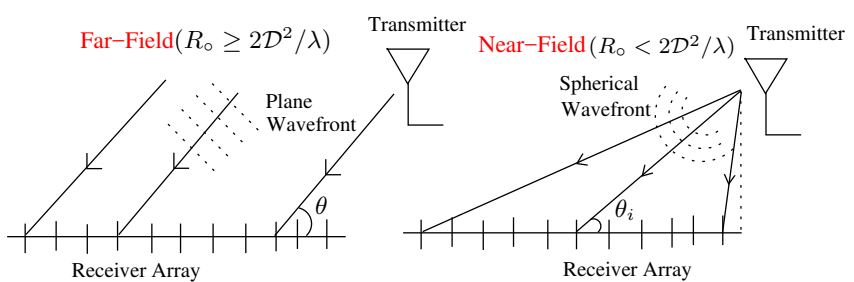

(a)

(b)

Fig. 3. Illustration of far-field and near-field models. (a) In the far-field scenario $\left(R_{o} \geq 2 \mathcal{D}^{2} / \lambda\right)$, the signal is assumed to strike the array as a planar wave. (b) the signal is assumed to be a spherical wavefront as $\left(R_{o}<2 \mathcal{D}^{2} / \lambda\right)$.

of the $n^{\text {th }}$ row and $m^{\text {th }}$ column entry of $\mathbf{H}_{\mathrm{ii}}^{(i)}$ is given by [16], [34]

$$
\left[\mathbf{H}_{\mathrm{ii}}{ }_{m n}^{(i)}=\frac{\rho}{r_{m n}} \exp \left(-j 2 \pi \frac{r_{m n}}{\lambda}\right),\right.
$$

where $\rho$ is the power normalization constant invoked for ensuring $\mathbb{E}\left[\left\|\mathbf{H}_{\mathrm{ii}}^{(i)}\right\|_{F}^{2}\right]=N_{t}^{(i)} N_{r}^{(i)}$, and $r_{m n}$ is the distance between the $m^{\text {th }}$ element of the transmitter and $n^{\text {th }}$ element of the the receiver, which is given in (9), where $\lambda, D$, and $\Theta$ are the wavelength, the distance between the first antenna elements of the transmitter and receiver arrays, and the angle between the arrays, respectively. The channel matrix $\mathbf{H}_{r p}$ of the reflected path is modeled as in (2) with the appropriate angle of departure and arrival. It is instructive to note that the near-field channel model considered in (8) is a simplified one as in [35]. It does not account for the mutual coupling effects between the antenna elements and the signal reflections.

For the system model in (1), the achievable sum rate of the system is given in (10), where the term $\mathbf{Z}_{i}^{-1}$ captures the SI power due to the transmitter of node $i$ plus noise power at the receiver of node $i$. Furthermore, $P^{(j)}$ and $P_{\mathrm{SI}}^{(i)}$ denotes the transmit power of node $j$ and the SI power of node $i$, respectively.

Remark 1: The employment of FD hybrid beamforming is more plausible in a downlink scenario, where the base station is receiving a signal from one user at an angle $\phi_{1}$ and simultaneously transmitting to another user at an angle $\phi_{2}$. In other words, the transmitter transmits its own signal in one beam and receives the signal from a user in a different beam, hence mitigating the SI.

In the next section, we design the beamformer, precoder and combiner matrices to suppress the interference, while preserving the signal dimensions.

\section{Transceiver Design Proposed for mmWaVe SYSTEMS}

In this section, we present our proposed design, where we first conceive the local optimal digital precoder and combiner matrices for the system model of (1). Then, we split the digital precoder obtained into an analog RF beamformer matrix $\mathbf{F}_{\mathrm{RF}}^{(j)}$ and a digital precoder matrix $\mathbf{F}_{\mathrm{BB}}^{(j)}$.

\section{A. Proposed Precoder and Combiner Design}

Consider the system model in (1), where only digital precoding and combining are employed, hence we set $\mathbf{F}^{(j)}=$ $\mathbf{F}_{\mathrm{RF}}^{(j)} \mathbf{F}_{\mathrm{BB}}^{(j)}$ and $\mathbf{W}^{H^{(i)}}=\mathbf{W}_{\mathrm{BB}}^{H^{(i)}} \mathbf{W}_{\mathrm{RF}}^{H^{(i)}}$. Then, the system model in (1) reduces to

$$
\mathbf{y}^{(i)}=\underbrace{\mathbf{W}^{H^{(i)}} \mathbf{H}_{j i} \mathbf{F}^{(j)} \mathbf{s}^{(j)}}_{\text {desired signal }}+\underbrace{\mathbf{W}^{H^{(i)}} \mathbf{H}_{\mathrm{SI}}^{(i)} \mathbf{F}^{(i)} \mathbf{s}^{(i)}}_{\text {SI }}+\underbrace{\mathbf{W}^{H^{(i)}} \mathbf{n}^{(i)}}_{\text {noise }} .
$$

In this section, we aim for designing the precoder matrix $\mathbf{F}$ for a fixed combiner matrix $\mathbf{W}$ and then design the combiner matrix $\mathbf{W}$ for a fixed precoder matrix $\mathbf{F}$. We carry out this process of optimization in iterations so that the effect of SI is minimized. The self-interference plus noise at the receiver node $i$ for fixed precoder matrix $\mathbf{F}^{(i)}$ is

$$
\mathbf{S} \mathbf{I}^{(i)}=\mathbf{W}^{H^{(i)}} \mathbf{H}_{\mathrm{SI}}^{(i)} \mathbf{F}^{(i)} \mathbf{s}^{(i)}+\mathbf{W}^{H^{(i)}} \mathbf{n}^{(i)},
$$

where the co-variance matrix $\mathbf{Q}^{(i)}$ of the interference plus noise at the receiver node $i$ is given by

$$
\mathbf{Q}^{(i)}=\operatorname{Tr}\left(\mathbf{W}^{H^{(i)}} \mathbf{R}^{(i)} \mathbf{W}^{(i)}\right),
$$

with $\mathbf{R}^{(i)}$ given by

$$
\mathbf{R}^{(i)}=P_{\mathrm{SI}}^{(i)} \mathbf{H}_{\mathrm{SI}}^{(i)} \mathbf{F}^{(i)}\left(\mathbf{H}_{\mathrm{SI}}^{(i)} \mathbf{F}^{(i)}\right)^{H}+\mathbf{I}_{n},
$$

and $\mathbf{I}_{n}$ is the noise power. Our objective is to design $\mathbf{W}$ and $\mathbf{F}$ so that we minimize the self-interference power $\mathbf{Q}^{(i)}$ at the receiver and simultaneously preserve the dimension of the signal space, i.e. $\operatorname{rank}\left(\mathbf{W}^{H^{(i)}} \mathbf{H}_{j i} \mathbf{F}^{(j)}\right)=N_{s}^{(j)}$.

Proposition 1: For the equality constrained optimization problem of

$$
\begin{aligned}
\min _{\mathbf{W}^{(i)}} & \operatorname{Tr}\left(\mathbf{W}^{H^{(i)}} \mathbf{R}^{(i)} \mathbf{W}^{(i)}\right) \\
\text { s.t. } & \mathbf{W}^{H^{(i)}} \mathbf{H}_{j i} \mathbf{F}^{(j)}=\alpha \mathbf{I}_{N_{s}}
\end{aligned}
$$

where $\mathbf{R}^{(i)}$ is a positive definite matrix $\left(\mathbf{R}^{(i)} \succ 0\right)$, the local optimal solution is given by

$$
\mathbf{W}_{\mathrm{opt}}^{(i)}=\alpha \mathbf{R}^{(i)^{-1}} \mathbf{H}_{j i} \mathbf{F}^{(j)}\left(\left(\mathbf{H}_{j i} \mathbf{F}^{(j)}\right)^{H} \mathbf{R}^{(i)^{-1}}\left(\mathbf{H}_{j i} \mathbf{F}^{(j)}\right)\right)^{-1}
$$

and $\alpha$ is the normalization constant expressed as

$$
\alpha=\frac{1}{\sqrt{\operatorname{Tr}\left(\mathbf{W}_{\mathrm{opt}}^{H(i)} \mathbf{W}_{\mathrm{opt}}^{(i)}\right)}} .
$$

Proof: The proof is relegated to Appendix A.

As seen from (16), $\mathbf{W}_{\text {opt }}^{(i)}$ is a function of $\mathbf{F}^{(j)}$, while our objective is to design $\mathbf{W}$ and $\mathbf{F}$ jointly. Hence, we opt for an iterative design, where we initially set $\mathbf{F}^{(j)}$ to the right singular vector of the channel matrix $\mathbf{H}_{j i}$ to get $\mathbf{W}_{\mathrm{opt}}^{(i)}$ and then having obtained the combiner matrix $\mathbf{W}^{(i)}$, we now proceed to design the precoder matrix $\mathbf{F}^{(j)}$ by considering the interference caused by the transmitter at the node $j$ to the local receiver.

The self-interference power imposed by the transmitter of 


$$
\begin{gathered}
r_{m n}=\sqrt{\left(\frac{D}{\tan (\Theta)}+(n-1) \frac{\lambda}{2}\right)^{2}+\left(\frac{D}{\sin (\Theta)}+(m-1) \frac{\lambda}{2}\right)^{2}-2\left(\frac{D}{\tan (\Theta)}+(n-1) \frac{\lambda}{2}\right)\left(\frac{D}{\sin (\Theta)}+(m-1) \frac{\lambda}{2}\right) \cos (\Theta),} \\
C=\sum_{j} \log _{2} \operatorname{det}\left(\mathbf{I}_{N_{s}}+\frac{P^{(j)}}{N_{\circ}} \mathbf{Z}_{i}^{-1}\left(\mathbf{F}_{\mathrm{RF}}^{(j)} \mathbf{F}_{\mathrm{BB}}^{(j)}\right)^{H} \mathbf{H}_{j i}^{H} \mathbf{W}_{\mathrm{BB}}^{(i)} \mathbf{W}_{\mathrm{RF}}^{(i)} \mathbf{W}_{\mathrm{BB}}^{H^{(i)}} \mathbf{W}_{\mathrm{RF}}^{H^{(i)}} \mathbf{H}_{j i} \mathbf{F}_{\mathrm{RF}}^{(j)} \mathbf{F}_{\mathrm{BB}}^{(j)}\right), j=1,2 ; i=1,2 ; j \neq i \\
\mathbf{Z}_{i}=P_{\mathrm{SI}}^{(i)} \mathbf{W}_{\mathrm{BB}}^{H^{(i)}} \mathbf{W}_{\mathrm{RF}}^{H^{(i)}} \mathbf{H}_{\mathrm{SI}}^{i} \mathbf{F}_{\mathrm{RF}}^{(i)} \mathbf{F}_{\mathrm{BB}}^{(i)}\left(\mathbf{W}_{\mathrm{BB}}^{H^{(i)}} \mathbf{W}_{\mathrm{RF}}^{H^{(i)}} \mathbf{H}_{\mathrm{SI}}^{i} \mathbf{F}_{\mathrm{RF}}^{(i)} \mathbf{F}_{\mathrm{BB}}^{(i)}\right)^{H}+\sigma^{2} \mathbf{W}_{\mathrm{BB}}^{H^{(i)}} \mathbf{W}_{\mathrm{RF}}^{H^{(i)}} \mathbf{W}_{\mathrm{BB}}^{(i)} \mathbf{W}_{\mathrm{RF}}^{(i)}
\end{gathered}
$$

node $j$ on the receiver of node $j$ is given by

$$
\mathbf{J}^{(j)}=\operatorname{Tr}\left(\mathbf{F}^{H^{(j)}} \mathbf{S}^{(j)} \mathbf{F}^{(j)}\right)
$$

where $\mathbf{S}^{(j)}$ is given by

$$
\mathbf{S}^{(j)}=\left(P_{\mathrm{SI}}^{(j)} \mathbf{W}^{H^{(j)}} \mathbf{H}_{\mathrm{SI}}^{(j)}\right)^{H}\left(\mathbf{W}^{H^{(j)}} \mathbf{H}_{\mathrm{SI}}^{(j)}\right)+\mathbf{I} .
$$

Similarly, our objective here is to minimize the selfinterference power $\mathbf{S}^{(j)}$ caused by the transmitter.

Thus, the constrained optimization problem is given by

$$
\begin{aligned}
& \min _{\mathbf{F}^{(j)}} \operatorname{Tr}\left(\mathbf{F}^{H^{(j)}} \mathbf{S}^{(j)} \mathbf{F}^{(j)}\right) \\
& \text { s.t. } \mathbf{W}^{H^{(i)}} \mathbf{H}_{j i} \mathbf{F}^{(j)}=\beta \mathbf{I}_{N_{s}} .
\end{aligned}
$$

By using Proposition 1, we obtain the local optimal solution as

$\mathbf{F}_{\mathrm{opt}}^{(j)}=\beta \mathbf{S}^{(j)^{-1}} \mathbf{H}_{j i}^{H} \mathbf{W}^{H^{(j)}}\left(\mathbf{W}^{H^{(j)}} \mathbf{H}_{j i} \mathbf{S}^{(j)^{-1}}\left(\mathbf{W}^{H^{(j)}} \mathbf{H}_{j i}\right)^{H}\right)^{-1}$

and $\beta$ is the normalization constant expressed as

$$
\beta=\frac{1}{\sqrt{\operatorname{Tr}\left(\mathbf{F}_{\mathrm{opt}}^{H(j)} \mathbf{F}_{\mathrm{opt}}^{(j)}\right)}} .
$$

This is an iterative process and it is continued until the convergence of the objective functions (15) and (20) is reached. The pseudo code for the algorithm is presented in Algorithm 1. The proof of convergence is presented in the next section.

In this paper, we have assumed that both the nodes 1 and 2 can simultaneously transmit and receive the data. However, in practice, given the power constraints, it is reasonable to assume that only the base station is equipped with a complex FD transceiver while the mobile station is half-duplex (HD). In this scenario, the mobile station does not experience any SI, hence the precoder for the mobile station can be designed by setting $\mathbf{H}_{\mathrm{SI}}=0$ in (19). However, the precoder and combiner weights for the base station can be obtained from Algorithm 1.

Remark 2: It is worth observing from (15) and (20) that the objective functions not only minimize the interference leakage into the desired receiver but also preserve the degrees

\footnotetext{
${ }^{4}$ The term $\mathbf{I}$ is added in (19) to ensure that the matrix $\mathbf{S}^{(j)}$ is invertible. The physical meaning of this is that it minimizes the norm of the precoder matrix. However, its effect is counteracted by $\beta$, which ensures that the transmit power is constant, i.e. $\left\|\mathbf{F}^{(j)} \mathbf{s}^{(j)}\right\|_{\mathrm{F}}^{2}=N_{s}$. Therefore, the identity matrix role in (19) is to ensure that $\mathbf{S}^{(j)}$ is invertible, but it has no influence on the design regardless of the SNR/INR. It can be removed if $\mathbf{S}^{(j)}$ is invertible in practice.
}

Algorithm 1 Proposed Design for Full Duplex HBF system

1: Initialize $\mathbf{F}^{(j)}$ to right singular vectors of the channel matrix $\mathbf{H}_{j i}, j=1,2 ; i=1,2 ; j \neq i$;

2: Compute the matrices $\mathbf{R}^{(i)}, i=1,2$;

3: Obtain $\mathbf{W}^{(i)}=$

$$
\mathbf{R}^{(i)^{-1}} \mathbf{H}_{j i} \mathbf{F}^{(j)}\left(\left(\mathbf{H}_{j i} \mathbf{F}^{(j)}\right)^{H} \mathbf{R}^{(i)^{-1}}\left(\mathbf{H}_{j i} \mathbf{F}^{(j)}\right)\right)^{-1} ;
$$

4: Then normalize $\mathbf{W}^{(i)}$ by multiplication factor

$$
\frac{1}{\sqrt{\operatorname{Tr}\left(\mathbf{W}_{\mathrm{opt}}^{H(i)} \mathbf{W}_{\mathrm{opt}}^{(i)}\right)}} ;
$$

5: Compute the matrices $\mathbf{S}^{(j)}, j=1,2$;

6: Obtain $\mathbf{F}^{(j)}=$

$$
\mathbf{H}_{j i}^{H} \mathbf{W}^{H(j)}\left(\mathbf{W}^{H(j)} \mathbf{H}_{j i} \mathbf{S}^{(j)^{-1}}\left(\mathbf{W}^{H(j)} \mathbf{H}_{j i}\right)^{H}\right)^{-1} ;
$$

7: Then normalize $\mathbf{F}^{(j)}$ by multiplication factor $\frac{1}{\sqrt{\operatorname{Tr}\left(\mathbf{F}_{\mathrm{opt}}^{H(j)} \mathbf{F}_{\mathrm{opt}}^{(j)}\right)}} ;$

8: Repeat steps from 2 until convergence of functions (15) and (20) is reached.

of freedom for the desired signal, which is formulated in the constraint. The physical significance of the constraints in both the objective functions of (15) and (20) is ensuring that the signal does not suffer from distortions. The proposed objective functions can also be interpreted as maximizing the SINR, where the numerator (signal fidelity) is kept unaltered while reducing the denominator (interference) [36].

\section{B. Convergence of the Proposed Design}

In this subsection, we present the convergence of the objective function in (15) and (20). We show that the value of the objective function decreases in every iteration and converges to its local optimum in a few iterations, which makes it more computationally efficient in practical system designs. Here, we provide the proof for the convergence of the proposed algorithm. The total self-interference plus noise power at all receivers is given by

$$
\begin{aligned}
\mathbf{Q} & =\sum_{i=1}^{2} \operatorname{Tr}\left(\mathbf{W}^{H^{(i)}} \mathbf{R}^{(i)} \mathbf{W}^{(i)}\right) \\
& =\sum_{i=1}^{2} \operatorname{Tr}\left(\mathbf{W}^{H^{(i)}}\left(\mathbf{H}_{\mathrm{SI}}^{(i)} \mathbf{F}^{(j)}\left(\mathbf{H}_{\mathrm{SI}}^{(i)} \mathbf{F}^{(j)}\right)^{H}+\mathbf{I}_{n}\right) \mathbf{W}^{(i)}\right)
\end{aligned}
$$




$$
=\sum_{i=1}^{2} \operatorname{Tr}\left(\mathbf{W}^{H^{(i)}} \mathbf{H}_{\mathrm{SI}}^{(i)} \mathbf{F}^{(j)}\left(\mathbf{H}_{\mathrm{SI}}^{(i)} \mathbf{F}^{(j)}\right)^{H} \mathbf{W}^{(i)}\right)+1 .
$$

Similarly, the self-interference plus noise power at node $j$ is given by

$$
\begin{aligned}
\mathbf{J} & =\sum_{j=1}^{2} \operatorname{Tr}\left(\mathbf{F}^{H^{(j)}} \mathbf{S}^{(j)} \mathbf{F}^{(j)}\right) \\
& =\sum_{j=1}^{2} \operatorname{Tr}\left(\mathbf{F}^{H^{(j)}}\left(\left(\mathbf{W}^{H^{(j)}} \mathbf{H}_{\mathrm{SI}}^{(j)}\right)^{H}\left(\mathbf{W}^{H^{(j)}} \mathbf{H}_{\mathrm{SI}}^{(j)}\right)+\mathbf{I}_{n}\right) \mathbf{F}^{(j)}\right)
\end{aligned}
$$$$
=\sum_{j=1}^{2} \operatorname{Tr}\left(\left(\mathbf{W}^{H^{(j)}} \mathbf{H}_{\mathrm{SI}}^{(j)} \mathbf{F}^{(j)}\right)^{H}\left(\mathbf{W}^{H^{(j)}} \mathbf{H}_{\mathrm{SI}}^{(j)} \mathbf{F}^{(j)}\right)\right)+1 .
$$

It is instructive to note that the objective functions in (23) and (26) are identical. Hence, W which minimizes (23) also minimizes (26) and similarly $\mathbf{F}$ that minimizes (26) also minimizes (23). Furthermore, the local optimal solutions of the objective functions (15) and (20) are given by Proposition 1, and they are sure to converge to the locally optimal solution as it is guaranteed by the Algorithm 1 of the proposed design and the value of the objective function decreases in every iteration and it is lower bounded by zero.

Fig. 4 shows the convergence of the objective function (15) for the proposed design. It can be seen from the figure that the objective function converges to a local optimum solution in just five iterations. Furthermore, since objective functions for designing precoder and combiner are the same, as proved in (23) and (26), Fig. 4 holds for (20).

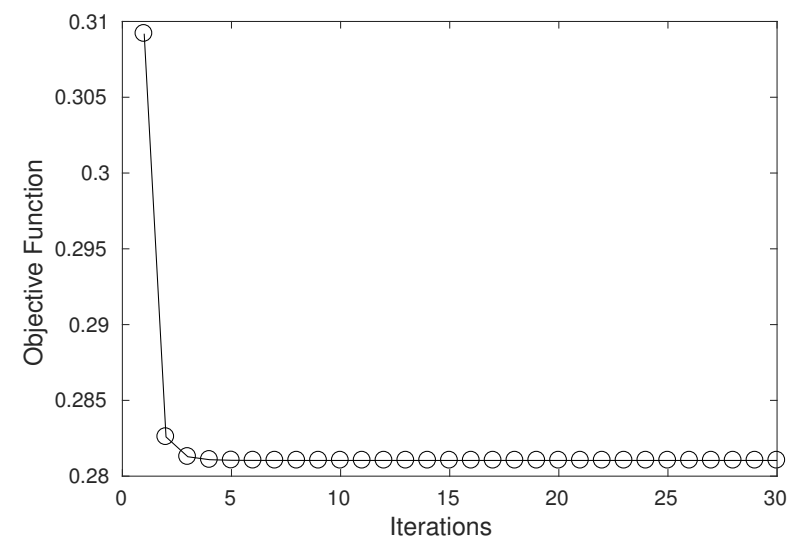

Fig. 4. Convergence of the objective function in (15) for the proposed design, $S I R=0 \mathrm{~dB}$ and $S N R=-10 \mathrm{~dB}$.

\section{Hybrid Beamforming for the Proposed Design}

In the previous sections, we presented the digital precoder and combiner design so as to minimize the SI. However, at the time of writing, the obtained resultant digital precoder and combiner design cannot be readily implemented in practice, since dedicating individual DACs/ADCs (RF chains) to each element of a large antenna array would impose a potentially excessive hardware complexity and a high power consumption. Therefore, to circumvent these challenges, typically a hybrid beamformer is employed, which has an analog RF beamformer and a baseband precoder. Thus, having obtained the fullydigital precoder solution, we now aim for decomposing the digital solution into a hybrid product, where we invoke the algorithm proposed in [37], which relies on the classic LS solution. Then similarly, the decomposition of the digital combiner matrix into an analog RF beamformer $\mathbf{W}_{\mathrm{RF}}$ and a baseband combiner $\mathbf{W}_{\mathrm{BB}}$ can be carried out.

It is important to note that decomposing the matrix $\mathbf{F}_{\mathrm{opt}}^{(j)}$ into the product of $\mathbf{F}_{\mathrm{RF}}^{(j)}$ and $\mathbf{F}_{\mathrm{BB}}^{(j)}$ is not straightforward because of the constraints imposed on the $\mathbf{F}_{\mathrm{RF}}^{(j)}$ matrix, where the entries in $\mathbf{F}_{\mathrm{RF}}^{(j)}$ must have constant modulus, otherwise we would have a power imbalance in the system. Therefore, we aim for finding the product of two matrices $\mathbf{F}_{\mathrm{RF}}$ and $\mathbf{F}_{\mathrm{BB}}$ which closely approximates the local optimal precoder $\mathbf{F}_{\mathrm{opt}}^{(j)}$. The optimization problem can be formulated as

$$
\begin{array}{r}
\min _{\mathbf{F}_{\mathrm{RF}}^{(j)}, \mathbf{F}_{\mathrm{BB}}^{(j)}}\left\|\mathbf{F}_{\mathrm{opt}}^{(j)}-\mathbf{F}_{\mathrm{RF}}^{(j)} \mathbf{F}_{\mathrm{BB}}^{(j)}\right\|_{F}^{2} . \\
\text { s.t. }\left|\mathbf{F}_{\mathrm{RF}}(m, n)\right|^{2}=1 .
\end{array}
$$

To solve the above optimization problem, we first fix $\mathbf{F}_{\mathrm{RF}}^{(j)}$ to the angle $\angle \mathbf{F}_{\text {opt }}^{(j)}$ and then find the sub optimal $\mathbf{F}_{\mathrm{BB}}^{(j)}$. This solution is then used for obtaining the unconstrained $\mathbf{F}_{\mathrm{RF}}^{(j)}$ in the next step.

Thus, the problem in (6) reduces to a two-stage optimization problem, which can be formulated as

$$
\begin{aligned}
& \mathbf{F}_{\mathrm{BB}_{k+1}}^{(j)} \triangleq \min _{\mathbf{F}_{\mathrm{BB}}^{(j)}}\left\|\mathbf{F}_{\mathrm{opt}}^{(j)}-\mathbf{F}_{\mathrm{RF}_{k}}^{(j)} \mathbf{F}_{\mathrm{BB}_{k}}^{(j)}\right\|_{F}^{2}, \\
& \mathbf{F}_{\mathrm{RF}_{k+1}}^{(j)} \triangleq \min _{\mathbf{F}_{\mathrm{RF}}^{(j)}}\left\|\mathbf{F}_{\mathrm{opt}}^{(j)}-\mathbf{F}_{\mathrm{RF}_{k}}^{(j)} \mathbf{F}_{\mathrm{BB}_{k}}^{(j)}\right\|_{F}^{2},
\end{aligned}
$$

where $k$ is the iteration index. Furthermore, Equations (31) and (32) represent a convex quadratically-constrained quadratic programming (QCQP) problem, whose solutions are given as [30], [37]

$$
\begin{aligned}
& \mathbf{F}_{\mathrm{BB}_{k+1}}^{(j)}=\left(\mathbf{F}_{\mathrm{RF}_{k}}^{H^{(j)}} \mathbf{F}_{\mathrm{RF}_{k}}^{(j)}\right)^{-1} \mathbf{F}_{\mathrm{RF}_{k}}^{H^{(j)}} \mathbf{F}_{\mathrm{opt}}^{(j)}, \\
& \mathbf{F}_{\mathrm{RF}_{k+1}}^{(j)}=\mathbf{F}_{\mathrm{opt}^{(j)}}^{\mathbf{F}_{\mathrm{BB}}^{H(j)}}\left(\mathbf{F}_{\mathrm{BB}_{k+1}}^{(j)} \mathbf{F}_{\mathrm{BB}}^{H(j)}\right)^{-1} .
\end{aligned}
$$

Since the $\mathbf{F}_{\mathrm{RF}_{k+1}}^{(j)}$ obtained in (34) is unconstrained, we apply the Proposition 2 [37] to obtain the constrained $\mathbf{F}_{\mathrm{RF}_{k+1}}^{(j)}$, where the magnitudes of the entries are constant.

Proposition 2 [37]: Suppose $\mathbf{A} \in \mathbb{C}^{N_{t} \times N_{s}}$ is expressed as $\mathbf{A}(m, n)=|\mathbf{A}(m, n)| e^{j \angle \mathbf{A}(m, n)}, \forall m, n$. Let $\mathcal{S}=\{\mathbf{B} \in$ $\left.\mathbb{C}^{N_{t} \times N_{s}}|\quad| \mathbf{B}(m, n) \mid=1 / \sqrt{N_{t}}, \forall m, n\right\}$ and

$$
\mathbf{U}^{\prime}=f(\mathbf{A}) \triangleq \arg \min _{\mathbf{U} \in \mathcal{S}}\|\mathbf{A}-\mathbf{U}\|_{\mathrm{F}}^{2}
$$

is the Euclidean projection of $\mathbf{A}$ onto the set $\mathcal{S}$. Then, $\mathbf{U}^{\prime}=$ $\frac{1}{\sqrt{N_{t}}} e^{<\mathbf{A}(m, n)}$.

Proof: We refer readers to [37].

Thus, $\mathbf{F}_{\mathrm{RF}_{k+1}}^{(j)}=f\left(\mathbf{F}_{\mathrm{opt}}^{(j)} \mathbf{F}_{\mathrm{BB}_{k+1}}^{H(j)}\left(\mathbf{F}_{\mathrm{BB}_{k+1}}^{(j)} \mathbf{F}_{\mathrm{BB}_{k+1}}^{H(j)}\right)^{-1}\right)$.

In other words, we set the magnitude of each entry in $\mathbf{F}_{\mathrm{RF}_{k+1}}^{(j)}$ 
to $1 / \sqrt{N_{t}^{(j)}}$ and the phase as $\angle \mathbf{F}_{\mathrm{RF}_{k+1}}^{(j)}$. A similar procedure is followed to obtain $\mathbf{W}_{\mathrm{RF}}$ and $\mathbf{W}_{\mathrm{BB}}$.

The pseudo-code for hybrid precoding is presented in Algorithm 2.

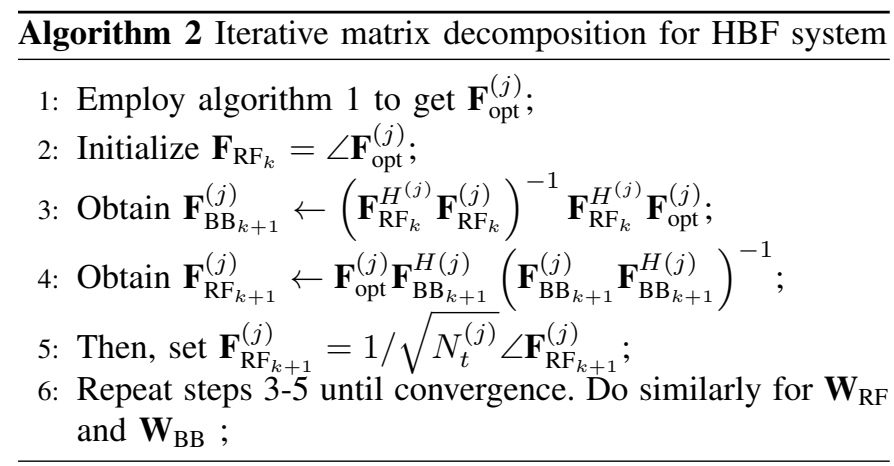

TABLE I. Simulation parameters

\begin{tabular}{lr}
\hline \hline Parameters & Values \\
\hline$N_{t}^{(1)}$ & 128 \\
$N_{t}^{(2)}$ & 128 \\
$N_{r}^{(1)}$ & 32 \\
$N_{r}^{(2)}$ & 32 \\
$N_{t_{1}}^{\mathrm{RF}}$ & 2 \\
$N_{t_{2}}^{\mathrm{RF}}$ & 2 \\
$N_{s}^{1}$ & 2 \\
$N_{s}^{2}$ & 2 \\
$\kappa[\mathrm{dB}]$ & 50 \\
$\phi$ & $\mathcal{U}(-\pi / 6, \pi / 6)$ \\
$\theta$ & $\mathcal{U}(-\pi / 6, \pi / 6)$ \\
Modulation & $\mathrm{QPSK}$ \\
\hline
\end{tabular}

Note that since the scope of the paper is not explicitly on the matrix decomposition of the HBF system, we have resorted to using the classic LS solutions. However approaches of [38][40] may also be invoked for matrix decomposition.

\section{Simulation Results}

In this section, we characterize the performance of the proposed design and compare it to that of eigen beamforming, where the left and right singular matrices of the corresponding channel are employed. We use the sum rate expression of (10) as the performance metric for our simulations. The parameters used in our simulations are summarized in Table I. The Rician factor in the SI channel model in (7) is set as high as $50 \mathrm{~dB}$ in our simulations, since the SI channel is LOS dominant.

Fig. 5 compares the sum rate of both the proposed design and of eigen beamforming, where the dominant eigen vectors are chosen as the precoder matrix. Furthermore, the curves shown in the figure are without hybrid beamforming, i.e. with the fully-digital beamforming. We also show in Fig. 5 the achievable sum rate, when the system is interference free. It is seen in Fig. 5 that for the signal-to-interference ratios (SIRs) of $-10 \mathrm{~dB}$ as well as $-30 \mathrm{~dB}$ and when $\Theta=0$ and $D=0$, the proposed design yields significant sum rate gains. Moreover, when the SIR is as low as $-30 \mathrm{~dB}$, the proposed design almost completely eliminates the interference and performs close to the system subjected to no interference. On the other hand, the performance of eigen beamforming is inferior to the proposed design by a large margin.

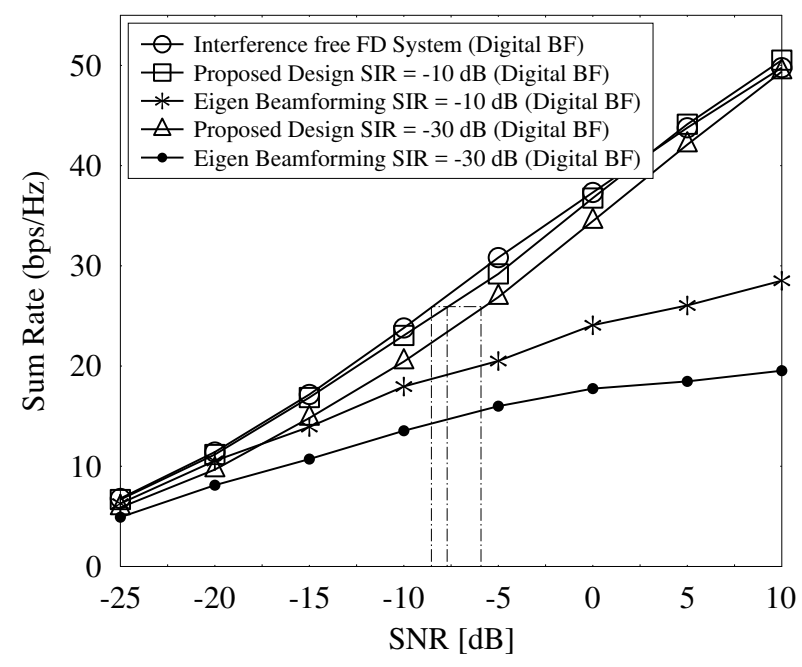

Fig. 5. Digital precoding of the proposed design for $S I R=-10$ $d B,-30 d B$. The distance between the transmit and receive antenna arrays is $D=0$ and the angle between the arrays is $\Theta=0^{\circ}$. The parameters of Table 1 are used.

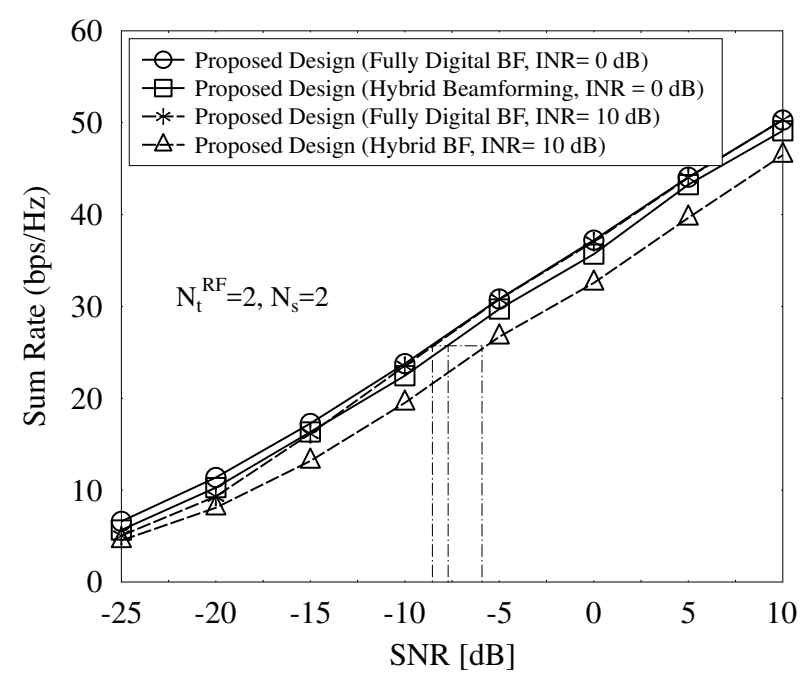

Fig. 6. Sum rate of hybrid beamforming relying on the proposed design for $I N R=0 \mathrm{~dB}$ as well as $10 \mathrm{~dB}$. The distance between the transmit and receive antenna arrays is $D=0$ and the angle between the arrays is $\Theta=10^{\circ}$. The parameters of Table 1 are used.

Fig. 6 shows the sum rate performance of the unconstrained (fully-digital) and constrained (hybrid beamforming) designs 
of the proposed algorithm. It can be seen from the figure that for the interference-to-noise ratio (INR) of $0 \mathrm{~dB}$ the performance of the fully-digital design and of the hybrid beamforming designs is similar. However, when the INR is increased to $10 \mathrm{~dB}$, the hybrid beamforming of the proposed design starts deviating from the fully-digital design. This is because of the constraint in the RF beamforming to have constant gain entries. The error in splitting the fully-digital matrix into hybrid beamforming matrices is amplified as the INR is increased.

The comparison of the sum rate of the proposed design and of the eigen beamforming using hybrid beamforming is shown in Fig. 7. The curves portrayed in the figure are simulated for SIRs $=-20 \mathrm{~dB}$ and $-25 \mathrm{~dB}$, when $\mathrm{D}=0$ and $\Theta=0^{\circ}$. It is seen that the proposed beamforming solution outperforms eigen beamforming by about $5 \mathrm{~dB}$ for both the SIRs considered.

To further examine the sum rate of both systems, Fig. 8 shows the sum rate of the proposed design for $I N R=10 \mathrm{~dB}$, $25 \mathrm{~dB}$. Since the residual self-interference in FD is significant in practice, an INR as high as $25 \mathrm{~dB}$ is considered for Fig. 8. It is again evident from the figure that for both the INRs of $10 \mathrm{~dB}$ and $25 \mathrm{~dB}$, the proposed design achieves better sum rate than the eigen beamforming based hybrid beamforming. The proposed design is capable of better mitigating the selfinterference and hence provides about $5 \mathrm{~dB}$ gain against the eigen beamforming system.

Fig. 9 (a) shows the achievable rate of both FD and HD systems for a $128 \times 32$ element MIMO. It is interesting to note that for higher levels of INR at low SNRs the HD mode provides better sum rate than the FD systems, which can be observed at the cross-over points in the Fig. 9 for INR levels of $10 \mathrm{~dB}$ and $15 \mathrm{~dB}$. Therefore, in system design, it is important to consider the operating region of FD and HD modes depending on the INR levels, especially at mmWave frequencies because of the dominant residual SI. Furthermore, it is instructive to observe that when the MIMO size is reduced to $64 \times 32$, the cross-over shifts further to right as observed in Fig. 9 (b). In other words, the $128 \times 32$ element MIMO provides higher beamforming gain with narrow beams to circumvent the SI when compared to that of $64 \times 32$ element MIMO. Thus, large antenna arrays may be employed for directional transmission to suppress the SI efficiently.

Additionally, the performance of the system also depends on the position of the transmit and receive antenna arrays. To study the system performance for both designs with respect to the transmit and receive antenna arrays placement, Fig. 10 shows the sum rate for different values of $D$. It is worth observing from the figure that as the distance $\mathrm{D}$ between the transmit and receive antenna is increased from $2 \lambda$ to $20 \lambda$, the performance of the system improves significantly. This is because for the increased separation, the self-interference would experience higher path loss, which results in passive interference suppression, thus a gain of about $5 \mathrm{~dB}$ is observed when $D$ is set to $20 \lambda$. Hence, superior performance is observed.

Another important parameter that affects the system's performance is the angle between the transmit and receive antenna arrays. Fig. 11 shows the performance of both system designs,

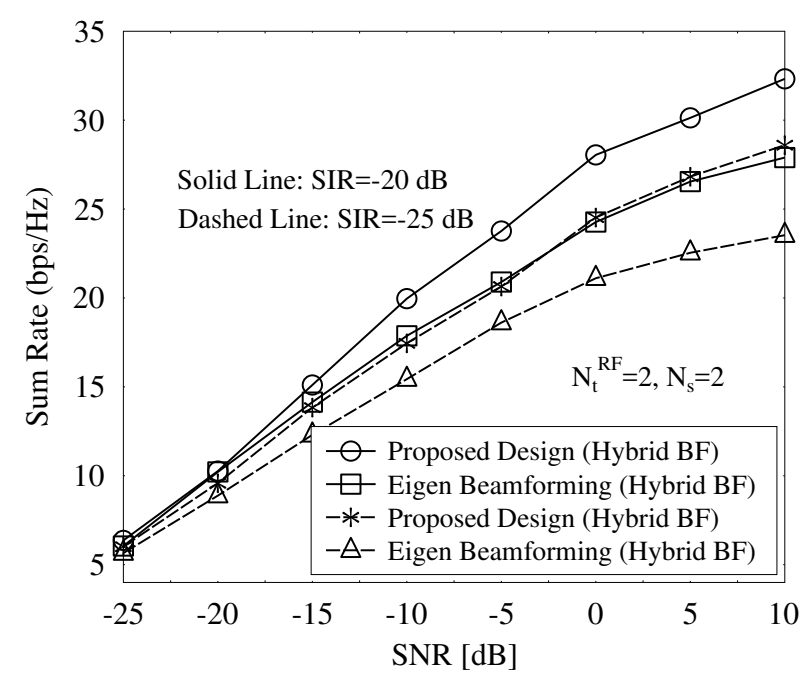

Fig. 7. Sum rate comparison of the proposed design and of eigen beamforming using hybrid beamforming for different SIRs. The distance between the transmit and receive antenna arrays is $D=0$ and the angle between the arrays is $\Theta=10^{\circ}$. The parameters of Table 1 are used.

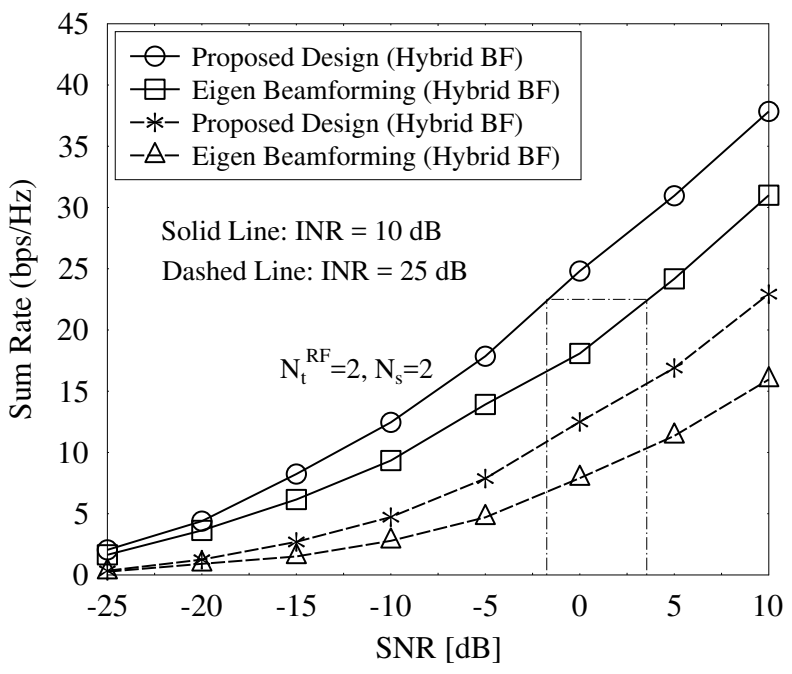

Fig. 8. Comparison of the sum rate of the proposed design and of eigen beamforming using hybrid beamforming for different INRs. The distance between the transmit and receive antenna arrays is $D=0$ and the angle between the arrays is $\Theta=0^{\circ}$. The parameters of Table 1 are used.

when the angle between the arrays is varied. It is seen that for both designs, the sum rate remains low when the angle between the arrays $\Theta=0^{\circ}, 180^{\circ}$ and $360^{\circ}$ and $\mathrm{D}=0$. This is expected, because when they share same antenna array (as $\Theta=0^{\circ}$ and $\mathrm{D}=0$ ), the SI would be high, which has 


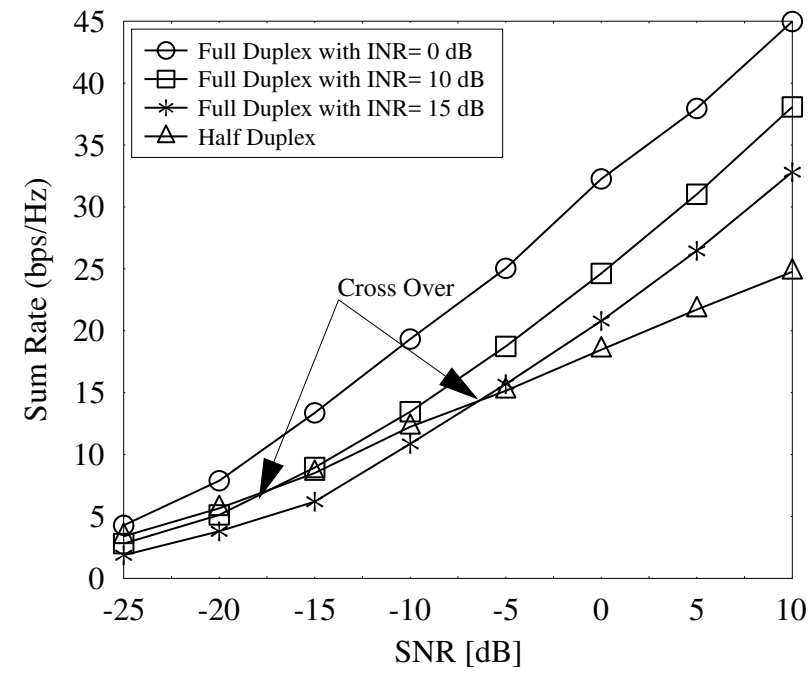

(a)

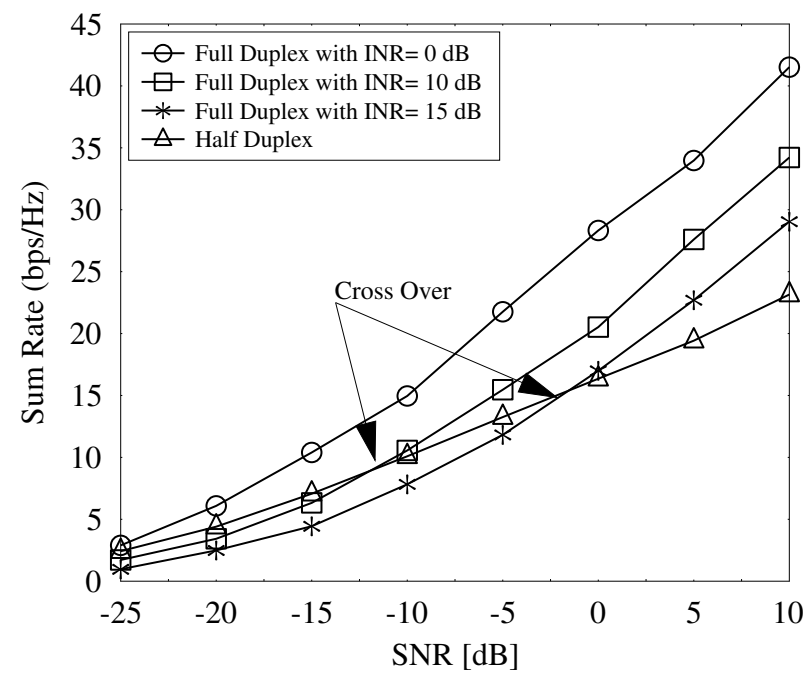

(b)

Fig. 9. Comparison of the sum rate of $F D$ and $H D$ modes at different SI levels - (a) $128 \times 32$ (b) $64 \times 32$. The distance between the transmit and receive antenna arrays is $D=0$ and the angle between the arrays is $\Theta=0^{\circ}$. The parameters of Table 1 are used.

detrimental effects on the system's performance. However, when the arrays are placed at different angles, the sum rate improves, because the angles of arrival and departure would be different, which makes the transmit and receive signal separation more efficiently.

Furthermore, in order to understand the effect of channel estimation error on the performance of the proposed design, we simulated the bit error rate (BER) performance of the proposed system, when considering perfect channel knowledge as well as erroneous CSI at the transmitter and receiver. Fig. 12 shows the BER curve for both perfect and imperfect CSI, when the precoder and combiner of Section III-C are employed. In

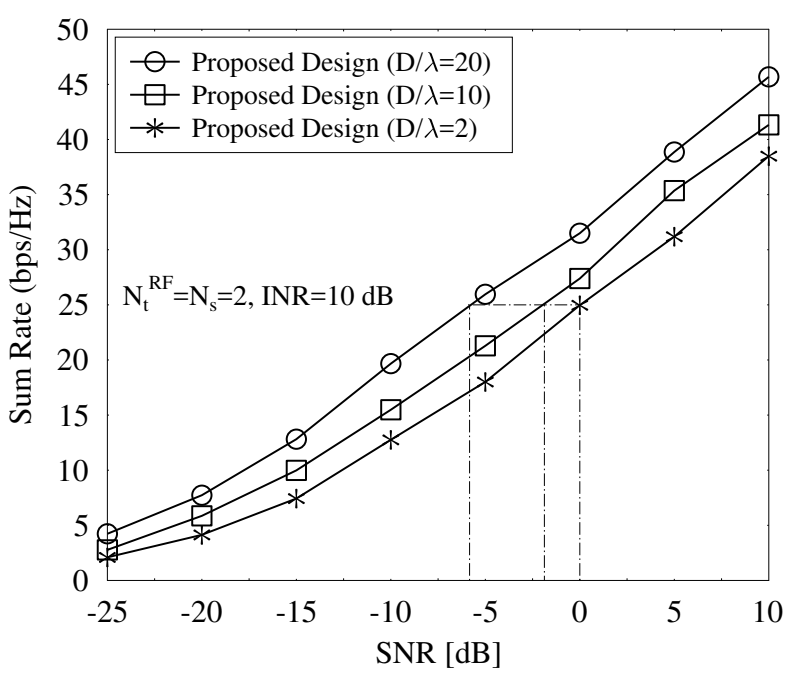

Fig. 10. Sum rate of the proposed design for different values of $D$ when $I N R=10 \mathrm{~dB}$ and angle between the transmit and receive antenna arrays $\Theta=0^{\circ}$.

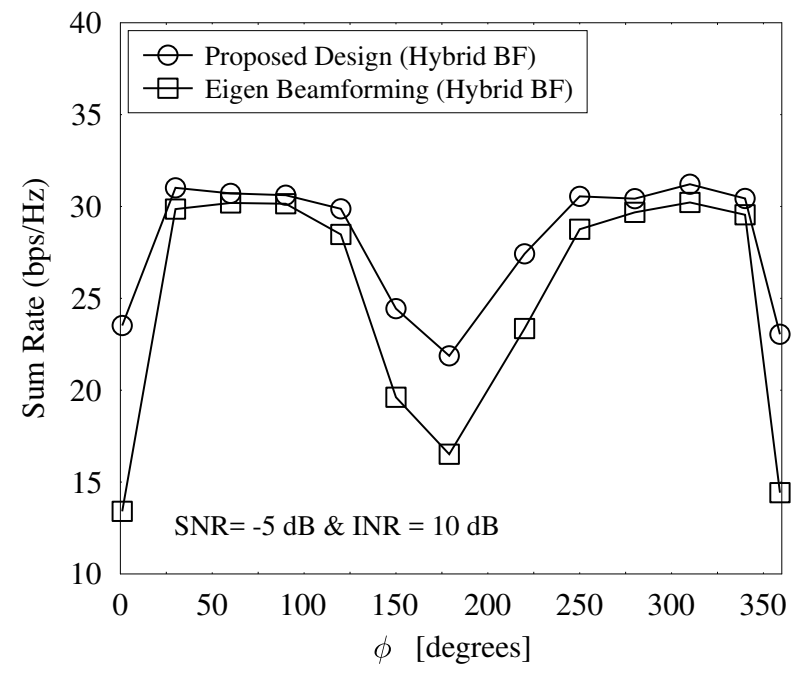

Fig. 11. Sum rate performance vs angle between the arrays when $D=0, S N R=-5 d B$ and $I N R=10 d B$.

this simulation the error in the channel estimate is assumed to be distributed as complex Gaussian with mean 0 and variance $\sigma_{h}^{2}$ i.e, $\sim \mathcal{C N}\left(0, \sigma_{h}^{2}\right)$ [41]. We have investigated the BER performance for $\sigma_{h}^{2}$ as low as 0.1 , and for $\sigma_{h}^{2}$ as high as 25 [41]. It can be seen from the Fig. 12 that there is a degradation in the performance of the BER when the channel is imperfect to that of when there is perfect CSI. It is instructive to note that the proposed designed is for maximizing the sum rate, which need not necessarily minimize the BER. Furthermore, the FD is conceived for capacity enhancement, not for BER- 


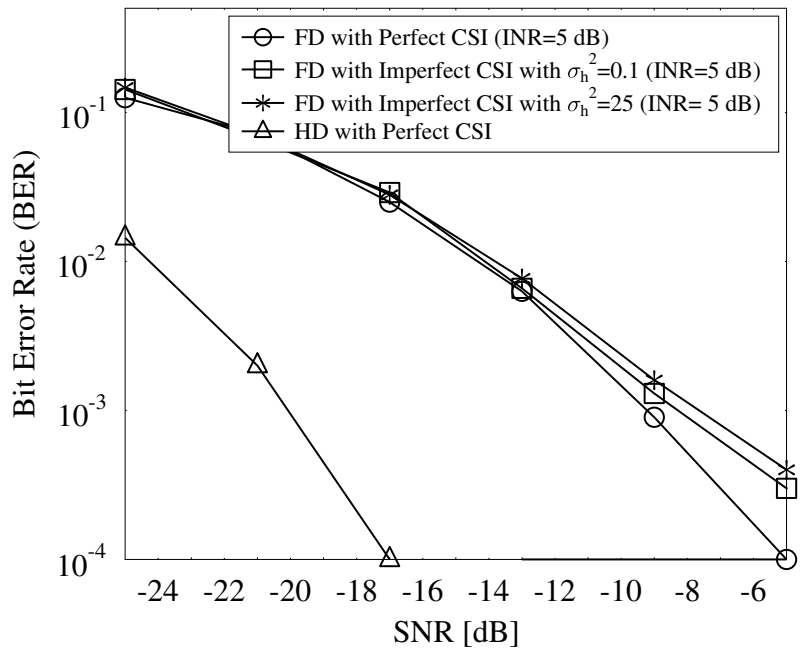

Fig. 12. BER of the proposed design for FD under imperfect CSI. To obtain this curve, a single stream with QPSK modulation is transmitted using $128 \times 32$ element MIMO. The parameters of Table 1 are used.

reduction. Although the uncoded BER performance of the FD may remain inferior to that of $\mathrm{HD}$ as in Fig. 12, the coded BER performance of the FD scheme is capable of approaching the capacity [42].

\section{CONCLUSIONS}

An iterative hybrid beamforming design was proposed for FD in mmWave communications, where the SI is cancelled by the joint design of beamformer weights in the RF and transmit precoder in the baseband. Our proposed design preserves the dimensions of the transmit signal, i.e. rank of the signal dimension is not affected, while suppressing the SI. We first obtain the full digital precoder/combiner matrices, and then decouple the obtained precoder matrix into RF beamformer matrix and baseband precoder matrix. Furthermore, we show that the algorithm converges in fewer iterations, which makes it computationally efficient in practical system design. Later in the paper, we demonstrate that our joint design is capable of suppressing the SI by a maximum of $30 \mathrm{~dB}$ higher than the signal power and performs similarly to the interference-free FD system.

\section{APPENDIX A}

\section{PROOF OF PROPOSITION 1}

Proof: The objective is to minimize the equality constrained problem in (15), while maintaining the signal dimensions. To solve the above optimization problem, we begin by forming the Lagrangian function given as (36).

$$
\mathcal{L}\left(\mathbf{W}^{(i)}, z\right)=\left(\mathbf{W}^{H^{(i)}} \mathbf{R}^{(i)} \mathbf{W}^{(i)}\right)+z\left(\mathbf{W}^{H^{(i)}} \mathbf{H}_{j i} \mathbf{F}^{(j)}-\mathbf{I}_{N_{s}}\right)
$$

Then, the Lagrangian conditions for this problem are

$$
\begin{aligned}
& \nabla_{\mathbf{W}_{\mathrm{opt}}^{H(i)}} \mathcal{L}=0 \\
& z^{*}\left(\mathbf{W}_{\mathrm{opt}}^{H(i)} \mathbf{H}_{j i} \mathbf{F}^{(j)}-\alpha \mathbf{I}_{N_{s}}\right)=0
\end{aligned}
$$

Explicitly (37) can be written as,

$$
\begin{aligned}
& \nabla_{\mathbf{W}_{\mathrm{opt}}^{H(i)}} \operatorname{Tr}\left(\mathbf{W}_{\mathrm{opt}}^{H(i)} \mathbf{R}^{(i)} \mathbf{W}_{\mathrm{opt}}^{(i)}\right) \\
& +z^{*} \nabla_{\mathbf{W}_{\mathrm{opt}}^{H(i)}}\left(\mathbf{W}_{\mathrm{opt}}^{H^{(i)}} \mathbf{H}_{j i} \mathbf{F}^{(j)}-\mathbf{I}_{N_{s}}\right)=0
\end{aligned}
$$

where $\nabla$ is the gradient operation and $z^{*}$ is the Lagrangian multiplier.

By taking the derivative with the respect to $\mathbf{W}^{H^{(i)}}$ in equation (39), we obtain

$$
\begin{aligned}
& \mathbf{R}^{(i)} \mathbf{W}_{\mathrm{opt}}^{(i)}+z \mathbf{H}_{j i} \mathbf{F}^{(j)}=0 \\
& \mathbf{W}_{\mathrm{opt}}^{(i)}=-\mathbf{R}^{(i)^{-1}} \mathbf{H}_{j i} \mathbf{F}^{(j)} z .
\end{aligned}
$$

Upon substituting $\mathbf{W}_{\mathrm{opt}}^{(i)}$ in (38), we get

$$
\begin{aligned}
& \left(-\mathbf{R}^{(i)^{-1}} \mathbf{H}_{j i} \mathbf{F}^{(j)} z\right)^{H} \mathbf{H}_{j i} \mathbf{F}^{(j)}=\alpha \mathbf{I}_{N_{s}} \\
& z=-\alpha\left(\left(\mathbf{H}_{j i} \mathbf{F}^{(j)}\right)^{H} \mathbf{R}^{(i)^{-1}} \mathbf{H}_{j i} \mathbf{F}^{(j)}\right)^{-1} .
\end{aligned}
$$

Hence,

$$
\mathbf{W}_{\text {opt }}^{(i)}=\alpha \mathbf{R}^{(i)^{-1}} \mathbf{H}_{j i} \mathbf{F}^{(j)}\left(\left(\mathbf{H}_{j i} \mathbf{F}^{(j)}\right)^{H} \mathbf{R}^{(i)^{-1}}\left(\mathbf{H}_{j i} \mathbf{F}^{(j)}\right)\right)^{-1}
$$

\section{REFERENCES}

[1] I. Hemadeh, K. Satyanarayana, M. El-Hajjar, and L. Hanzo, "Millimeterwave communications: Physical channel models, design considerations, antenna constructions and link-budget," IEEE Commun. Surveys Tuts., vol. PP, no. 99, pp. 1-1, 2018.

[2] D. Bharadia, E. McMilin, and S. Katti, "Full duplex radios," $A C M$ SIGCOMM, vol. 43, no. 4, pp. 375-386, 2013.

[3] E. Everett, M. Duarte, C. Dick, and A. Sabharwal, "Empowering fullduplex wireless communication by exploiting directional diversity," in Proc. ASILOMAR, 2011, pp. 2002-2006.

[4] Z. Zhang, K. Long, A. V. Vasilakos, and L. Hanzo, "Full-duplex wireless communications: Challenges, solutions, and future research directions," Proc. IEEE, vol. 104, no. 7, pp. 1369-1409, July 2016.

[5] Y. Niu et al., "A survey of millimeter wave (mmwave) communications for 5G: Opportunities and challenges," CoRR, vol. abs/1502.07228, 2015.

[6] G. Maccartney, M. Samimi, and T. Rappaport, "Exploiting directionality for millimeter-wave wireless system improvement," in Proc. ICC, June 2015, pp. 2416-2422.

[7] B. Le et al., "Analog-to-digital converters," IEEE Signal Process. Mag., vol. 22 , no. 6 , pp. 69-77, 2005.

[8] S. Han et al., "Large-scale antenna systems with hybrid analog and digital beamforming for millimeter wave 5G," IEEE Commun. Mag., vol. 53, no. 11, pp. 186-194, 2015.

[9] K. Satyanarayana, M. El-Hajjar, P. Kuo, A. Mourad, and L. Hanzo, "Dual-function hybrid beamforming and transmit diversity aided millimeter wave architecture," IEEE Trans. Veh. Technol., vol. 67, no. 3, pp. 2798-2803, March 2018.

[10] O. E. Ayach, S. Rajagopal, S. Abu-Surra, Z. Pi, and R. W. Heath, "Spatially sparse precoding in millimeter wave MIMO systems," IEEE Trans. Wireless Commun., vol. 13, no. 3, pp. 1499-1513, March 2014.

[11] A. Alkhateeb and R. W. Heath, "Frequency selective hybrid precoding for limited feedback millimeter wave systems," IEEE Trans. Commun., vol. 64, no. 5, pp. 1801 - 1818, April 2016.

[12] M. E. Eltayeb et al., "Opportunistic beam training with analog/digital codebooks for mmwave systems," in Proc. GlobalSIP, Dec. 2015. 
[13] J. C. Chen, "Hybrid beamforming with discrete phase shifters for millimeter-wave massive MIMO systems," IEEE Trans. Veh. Technol. vol. 66, no. 8, pp. 7604-7608, Aug 2017.

[14] A. Sahai, G. Patel, C. Dick, and A. Sabharwal, "On the impact of phase noise on active cancelation in wireless full-duplex," IEEE Trans. Veh. Technol., vol. 62, no. 9, pp. 4494-4510, Nov 2013.

[15] M. Duarte and A. Sabharwal, "Full-duplex wireless communications using off-the-shelf radios: Feasibility and first results," in Proc. ASILOMAR, 2010, pp. 1558-1562.

[16] X. Liu, Z. Xiao, L. Bai, J. Choi, P. Xia, and X.-G. Xia, "Beamforming based full-duplex for millimeter-wave communication," Sensors, vol. 16, no. 7, 2016. [Online]. Available: http://www.mdpi.com/1424$8220 / 16 / 7 / 1130$

[17] A. Sabharwal, P. Schniter, D. Guo, D. W. Bliss, S. Rangarajan, and R. Wichman, "In-band full-duplex wireless: Challenges and opportunities," IEEE J. Sel. Areas Commun., vol. 32, no. 9, pp. 1637-1652, Sept 2014.

[18] M. Duarte, C. Dick, and A. Sabharwal, "Experiment-driven characterization of full-duplex wireless systems," IEEE Trans. Wireless Commun., vol. 11, no. 12, pp. 4296-4307, December 2012.

[19] Z. Xiao, P. Xia, and X. G. Xia, "Full-duplex millimeter-wave communication," IEEE Wireless Commun., vol. 24, no. 6, pp. 136-143, Dec 2017.

[20] T. Riihonen, S. Werner, and R. Wichman, "Mitigation of loopback selfinterference in full-duplex MIMO relays," IEEE Trans. Signal Process., vol. 59, no. 12, pp. 5983-5993, Dec 2011.

[21] Y. Liu, X. G. Xia, and H. Zhang, "Distributed linear convolutional space-time coding for two-relay full-duplex asynchronous cooperative networks," IEEE Trans. Wireless Commun., vol. 12, no. 12, pp. 64066417, December 2013.

[22] I. Krikidis, H. A. Suraweera, P. J. Smith, and C. Yuen, "Full-duplex relay selection for amplify-and-forward cooperative networks," IEEE Trans. Wireless Commun., vol. 11, no. 12, pp. 4381-4393, December 2012.

[23] L. Li, C. Dong, L. Wang, and L. Hanzo, "Spectral-efficient bidirectional decode-and-forward relaying for full-duplex communication," IEEE Trans. Veh. Technol., vol. 65, no. 9, pp. 7010-7020, Sept 2016.

[24] C. T. Lin, F. S. Tseng, and W. R. Wu, "MMSE transceiver design for full-duplex MIMO relay systems," IEEE Trans. Veh. Technol., vol. 66, no. 8, pp. 6849-6861, Aug 2017.

[25] D. W. K. Ng, E. S. Lo, and R. Schober, "Dynamic resource allocation in MIMO-OFDMA systems with full-duplex and hybrid relaying," IEEE Trans. Commun., vol. 60, no. 5, pp. 1291-1304, May 2012.

[26] S. Wang, Y. Liu, W. Zhang, and H. Zhang, "Achievable rates of fullduplex massive mimo relay systems over rician fading channels," IEEE Trans. Veh. Technol., vol. 66, no. 11, pp. 9825-9837, Nov 2017.

[27] B. P. Day, A. R. Margetts, D. W. Bliss, and P. Schniter, "Full-duplex mimo relaying: Achievable rates under limited dynamic range," IEEE $J$. Sel. Areas Commun., vol. 30, no. 8, pp. 1541-1553, September 2012.

[28] E. Everett, A. Sahai, and A. Sabharwal, "Passive self-interference suppression for full-duplex infrastructure nodes," IEEE Trans. Wireless Commun., vol. 13, no. 2, pp. 680-694, February 2014.

[29] T. Snow, C. Fulton, and W. J. Chappell, "Transmit-receive duplexing using digital beamforming system to cancel self-interference," IEEE Trans. Micro. Theory Techn., vol. 59, no. 12, pp. 3494-3503, Dec 2011.

[30] S. Boyd and L. Vandenberghe, Convex Optimization. New York, NY, USA: Cambridge University Press, 2004.

[31] T. Riihonen, S. Werner, and R. Wichman, "Spatial loop interference suppression in full-duplex MIMO relays," in Proc. ASILOMAR, Nov 2009 , pp. 1508-1512.

[32] M. K. Samimi and T. S. Rappaport, "3-D millimeter-wave statistical channel model for 5G wireless system design," IEEE Trans. Micro. Theory Techn., vol. 64, no. 7, pp. 2207-2225, July 2016.

[33] F. Wu, S. Shao, and Y. Tang, "Near-field self-interference cancellation and quality of service multicast beamforming in full-duplex," pp. 9902 - 9902 - 10, 2016. [Online]. Available: http://dx.doi.org/10.1117/12.2262058

[34] J.-S. Jiang and M. A. Ingram, "Spherical-wave model for short-range MIMO," IEEE Trans. Commun., vol. 53, no. 9, pp. 1534-1541, Sept 2005.

[35] Y.-P. Liao, H. Han, and Q. Guo, "Design of robust near-field multi-beam forming based on improved LCMV algorithm," J. Inf. Hiding Multimed. Signal Process, vol. 6, pp. 783-791, 2015.

[36] E. Antonio-Rodrguez, R. Lpez-Valcarce, T. Riihonen, S. Werner, and R. Wichman, "Sinr optimization in wideband full-duplex mimo relays under limited dynamic range," in n Proc. SAM Signal Process. Workshop, June 2014, pp. 177-180.
[37] H. Ghauch, T. Kim, M. Bengtsson, and M. Skoglund, "Subspace estimation and decomposition for large millimeter-wave MIMO systems," IEEE J. Sel. Topics Sig. Proc., vol. 10, no. 3, pp. 528-542, April 2016.

[38] X. Yu, J. C. Shen, J. Zhang, and K. B. Letaief, "Alternating minimization algorithms for hybrid precoding in millimeter wave MIMO systems," IEEE J. Sel. Topics Sig. Proc., vol. 10, no. 3, pp. 485-500, April 2016.

[39] L. He, J. Wang, and J. Song, "On generalized spatial modulation aided millimeter wave MIMO: Spectral efficiency analysis and hybrid precoder design," IEEE Trans. Wireless Commun., vol. 16, no. 11, pp. 7658-7671, Nov 2017.

[40] C. E. Chen, "An iterative hybrid transceiver design algorithm for millimeter wave MIMO systems," IEEE Wireless Commun. Lett., vol. 4, no. 3, pp. 285-288, June 2015.

[41] P. Aquilina and T. Ratnarajah, "Performance analysis of IA techniques in the MIMO IBC with imperfect CSI," IEEE Trans. Commun., vol. 63 , no. 4, pp. 1259-1270, April 2015.

[42] M. El-Hajjar and L. Hanzo, "Exit charts for system design and analysis," IEEE Commun. Surveys Tuts., vol. 16, no. 1, pp. 127-153, First 2014.

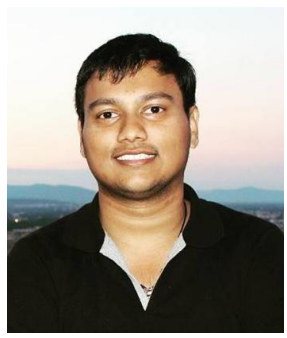

K. Satyanarayana (www.satyanarayana.xyz) received his B. Tech. degree in Electrical Engineering from Indian Institute of Technology Madras, India, in 2014. During Jul'14-Aug'15, he worked as a research assistant at Indian Institute of Science, Bangalore. Currently, Satya is a research scholar in Wireless Communications at the University of Southampton in liaison with InterDigital Europe, London, UK. His research interests include millimeter wave communications, hybrid beamforming, with an emphasis on transceiver algorithms for wireless communication systems and multi-functional MIMO.

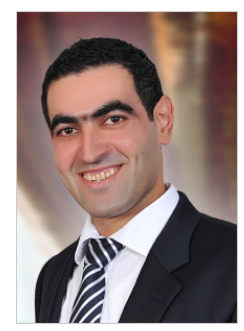

Mohammed El-Hajjar is an Associate Professor in the department of Electronics and Computer Science in the University of Southampton. He received his $\mathrm{PhD}$ in Wireless Communications from the University of Southampton, UK in 2008. Following the $\mathrm{PhD}$, he joined Imagination Technologies as a design engineer, where he worked on designing and developing Imaginations multi-standard communications platform, which resulted in three patents. $\mathrm{He}$ is the recipient of several academic awards and has published a Wiley-IEEE book and in excess of 80 journal and conference papers. Mohammed's research interests include the design of intelligent and energy-efficient transceivers, MIMO, millimeter wave communications, cross-layer optimization for large-scale networks and Radio over fiber network design.

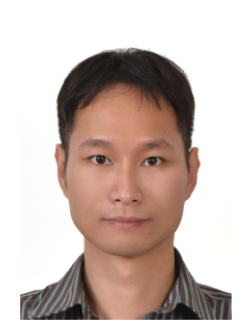

Ping-Heng Kuo is a staff engineer at InterDigital Europe, London, UK. He is currently involved in the European Horizon 2020 5G-PPP Phase-1 and Phase2 projects including $5 \mathrm{G}-$ Crosshaul and 5G-Coral, as well as additional collaborative projects in the UK researching pre-standard radio access technologies for $5 \mathrm{G}$ and beyond. Prior to joining InterDigital in 2016, he has been a wireless communications technologies research engineer and a 3GPP RAN1 delegate at Industrial Technology Research Institute (ITRI) in Taiwan for 7 years, focusing on advanced physical layer technologies such as CoMP and Massive-MIMO schemes. Ping-Heng also held a visiting scholar position at Harvard University in 2014, and he has served as an editor of Industry Perspectives Column for IEEE Wireless Communications Magazine in 2015. He received his Ph. D. from University of Canterbury, New Zealand, and conducted an internship at Samsung Advanced Institute of Technology (SAIT) in Korea. Ping-Heng is the main inventor of 8 granted US patents and has authored more than 20 peer-reviewed conference/journal papers. 


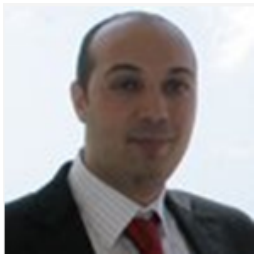

Alain A. M. Mourad holds a $\mathrm{PhD}$ degree in Telecommunications from ENST Bretagne in France. He has over 15 years experience in the wireless networks industry. He is currently leading the research and development of Next Generation Radio Access Networks at InterDigital International Labs (London, Berlin, Seoul). Prior to joining InterDigital, Dr. Mourad was a Principal Engineer at Samsung Electronics R\&D (UK) and previously a Senior Engineer at Mitsubishi Electric R\&D Centre Europe (France). Throughout his career, Dr. Mourad has been active in the research and standardization of recent communication networks $(5 \mathrm{G} / 4 \mathrm{G} / 3 \mathrm{G})$ and broadcasting systems (ATSC 3.0/DVB-NGH/DVBT2). He has held various leadership roles in the industry, invented over 35 granted patents and several other patent applications, and authored over 50 peer-reviewed publications. He received the Inventor of the Year Award from Samsung Electronics R\&D (UK) twice in 2012 and 2013, and in 2016 InterDigital Innovation Award for the idea, creation, and execution of InterDigital Europe.

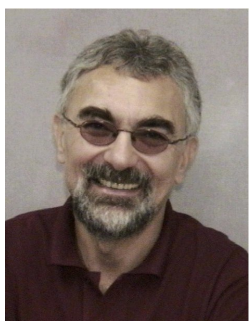

Lajos Hanzo (http://www-mobile.ecs.soton.ac.uk) FREng, F'04, FIET, Fellow of EURASIP, received his 5-year degree in electronics in 1976 and his doctorate in 1983 from the Technical University of Budapest. In 2009 he was awarded an honorary doctorate by the Technical University of Budapest and in 2015 by the University of Edinburgh. In 2016 he was admitted to the Hungarian Academy of Science. During his 40-year career in telecommunications he has held various research and academic posts in Hungary, Germany and the UK. Since 1986 he has been with the School of Electronics and Computer Science, University of Southampton, UK, where he holds the chair in telecommunications. He has successfully supervised $112 \mathrm{PhD}$ students, co-authored 18 John Wiley/IEEE Press books on mobile radio communications totalling in excess of 10000 pages, published $1800+$ research contributions at IEEE Xplore, acted both as TPC and General Chair of IEEE conferences, presented keynote lectures and has been awarded a number of distinctions. Currently he is directing a 60 -strong academic research team, working on a range of research projects in the field of wireless multimedia communications sponsored by industry, the Engineering and Physical Sciences Research Council (EPSRC) UK, the European Research Council's Advanced Fellow Grant and the Royal Society's Wolfson Research Merit Award. He is an enthusiastic supporter of industrial and academic liaison and he offers a range of industrial courses. He is also a Governor of the IEEE ComSoc and VTS. During 2008 - 2012 he was the Editor-in-Chief of the IEEE Press and a Chaired Professor also at Tsinghua University, Beijing. For further information on research in progress and associated publications please refer to http://www-mobile.ecs.soton.ac.uk 